\title{
Exact and Approximation Algorithms for Optimal Equipment Selection in Deploying In- Building Distributed Antenna Systems
}

\author{
David Adjiashvili, Sandro Bosio, Yuan Li and Di Yuan
}

Linköping University Post Print

Tweet

N.B.: When citing this work, cite the original article.

C2015 IEEE. Personal use of this material is permitted. However, permission to reprint/republish this material for advertising or promotional purposes or for creating new collective works for resale or redistribution to servers or lists, or to reuse any copyrighted component of this work in other works must be obtained from the IEEE.

David Adjiashvili, Sandro Bosio, Yuan Li and Di Yuan, Exact and Approximation Algorithms for Optimal Equipment Selection in Deploying In-Building Distributed Antenna Systems, 2015, IEEE Transactions on Mobile Computing, (14), 4, 702-713.

http://dx.doi.org/10.1109/TMC.2014.2331976

Postprint available at: Linköping University Electronic Press

http://urn.kb.se/resolve?urn=urn:nbn:se:liu:diva-117649 


\title{
Exact and Approximation Algorithms for Optimal Equipment Selection in Deploying In-Building
}

\section{Distributed Antenna Systems}

\author{
David Adjiashvili ${ }^{1}$, Sandro Bosio ${ }^{1}$, Yuan $\mathrm{Li}^{2}$, and Di Yuan ${ }^{3}$ \\ ${ }^{1}$ Institute for Operations Research, ETH Zürich, Switzerland \\ ${ }^{2}$ Department of Electrical and Information Technology, Lund University, Sweden \\ ${ }^{3}$ Department of Science and Technology, Linköping University, Sweden \\ Emails: \{david.adjiashvili, sandro.bosio\}@ifor.math.ethz.ch,yuan.li@eit.lth.se,di.yuan@liu.se
}

\begin{abstract}
We consider a combinatorial optimization problem in passive In-Building Distributed Antenna Systems (IB-DAS) deployment for indoor mobile broadband service. These systems have a tree topology, in which a central base station is connected to a number of antennas located at tree leaves via cables represented by the tree edges. Each inner node corresponds to a power equipment, of which the available types differ in the number of output ports and/or by power gain at the ports. This paper focuses on the equipment selection problem that amounts to, for a given passive DAS tree topology, selecting a power equipment type for each inner node and assigning the outgoing edges of the node to the equipment ports. The performance metric is the power deviation at the antennas from the target values. We consider as objective function the minimization of either the total or the largest power deviation over all antennas. Our contributions are the development of exact pseudo-polynomial time algorithms and (additive) fully-polynomial
\end{abstract}


time approximation schemes for both objectives. Numerical results are provided to illustrate the algorithms. We also extend some results to account for equipment cost.

Keywords - Wireless Communication, Combinatorial Algorithms, Trees

\section{Introduction}

Along the evolution of mobile communication technologies and user applications, in-building demand on network access is experiencing a substantial increase. Today, up to $80 \%$ of the mobile network traffic is generated by in-building users [9]. The trend is expected to escalate with the introduction of Long Term Evolution (LTE). Hence deploying dedicated systems to provide in-building coverage and capacity $[5,12]$ is a key step towards ubiquitous service.
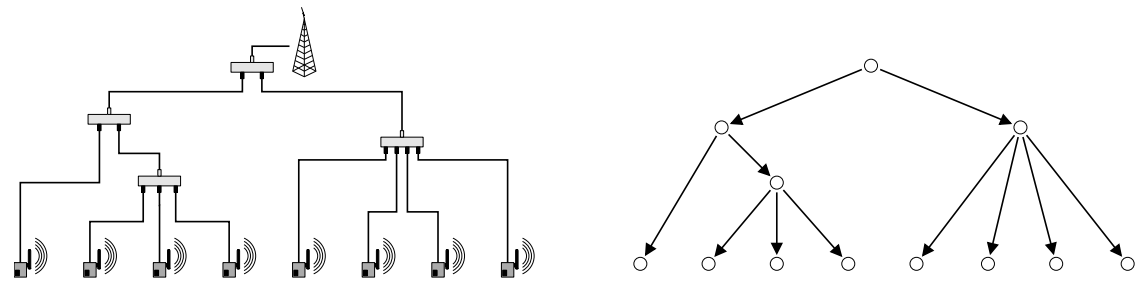

Figure 1: Schematic illustration of a P-DAS (left), with a base station connected to the antennas by means of coaxial cables and power splitters and taps. The resulting network topology is the rooted tree depicted on the right.

Deploying a dedicated in-building system requires performance engineering. The goal is to optimize the in-building network for high bandwidth and resource efficiency, while avoiding performance degradation to spectrum-shared outdoor systems [13, 22]. One of the emerging solutions for serving indoor traffic is the use of In-Building Distributed Antenna Systems (IBDAS). In this paper, we consider passive DAS (P-DAS) that does not require active electronic components. A typical P-DAS consists of a central base station (BS) connected to a number of antennas with a tree topology, as depicted in Figure 1. The BS corresponds to the tree root, and is the power source of all the antennas, which are the tree leaves. Each non-leaf node represents the location of a power equipment (a splitter or $\operatorname{tap}^{1}$ ), which divides the incoming

\footnotetext{
${ }^{1}$ The technical difference between a splitter and a tap is that splitters divide the incoming gain equally among the outgoing ports, while taps have uneven gain distributions.
} 
signal over two or more output ports. The connections between the nodes, i.e., the edges of the tree, are implemented using cables. Given a tree topology, the output power of an antenna can be computed from the output power of the BS (in logarithmic scale) by subtracting the signal loss over each edge and inner node in the unique path from the BS to the antenna. The signal loss over an edge is determined by the cable type and length. The signal loss due to an inner node is determined by the corresponding equipment type, and, if the equipment's ports have non-uniform power coefficients, by the output port used.

The output powers of the antennas form a key performance consideration. High output powers improve coverage and, to some extent, capacity. From an interference viewpoint, however, excessive output power should be avoided [22]. Typically, target powers of antennas are specified based on this trade-off, taking into account building characteristics and locations of user demand [3]. It may not be feasible to meet exactly the target powers, however, because there is no active electronic component in P-DAS, and consequently the actual output power is determined by topology and equipment selection for the inner nodes. The resulting performance metric is the power deviation at each antenna, that is, the absolute difference between the output power and the target power. On the cost side, the performance considerations include the expenditure of introducing and installing antennas and cable connections.

There are many design and optimization variables for radio access and infrastructure support for deploying and operating a P-DAS system. At the infrastructure side, the decisions include topology and equipment selection. In this paper, we focus on the P-DAS equipment selection problem, motived by the following facts.

- First, solving the problem arises in a decomposition approach in P-DAS deployment, namely topology planning followed by equipment selection. The approach is justified by the high complexity of the joint problem, for which scalability is prohibiting for obtaining reasonable solutions (by, e.g., integer programming [9]) for large-scale scenarios. Indeed, current DAS planning tools, such as [15], do follow the decomposition approach. In this case, the optimization process consists in generating and evaluating a large number of 
candidate topology solutions, for each of which equipment selection has to be carried out. For such planning tools, time-efficient solution of equipment selection is essential.

- Second, and more importantly, equipment selection is the major performance engineering instrument for re-optimization in the operational phase of P-DAS. A P-DAS has to undergo re-optimization for adapting to changes in user location and demand profile, resulting in adjustments of the antenna power targets. Re-optimization must not be costly, as it may have to take place rather frequently. On the infrastructure side, changing the topology (i.e., re-wiring cables) for the new power targets is not a preferable solution as it incurs high cost expenditure. In contrast, equipment re-selection and reconfiguration is much more apt to re-optimization, because re-installing equipment at the present locations and connecting their ports require little effort.

By the above motivations, the equipment selection problem for IB-DAS deserves a thorough study in its own right. To the best of our knowledge, there is no tractability, approximation, or algorithmic results for this problem, toward which the current paper contributes.

The input to the equipment selection problem consists of the following three parts.

- network topology in form of a rooted tree, where the root is the BS, and the leaves are antennas;

- power specification, namely BS power and the target power at each antenna;

- a set of compatible power equipment types for each inner node.

Given the input above, our optimization task is to select one equipment type for each inner node, and assign the equipment's ports to the outgoing edges connecting the node to its children in the tree. The performance objective, as was mentioned earlier, is the deviation of the antenna power, which is induced by equipment selection, from the target values. We consider both the minimization of the maximum (min-max) and the total (min-sum) power 


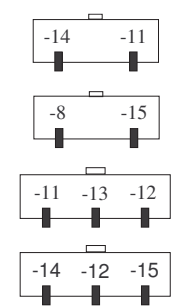

(a) Equipment types

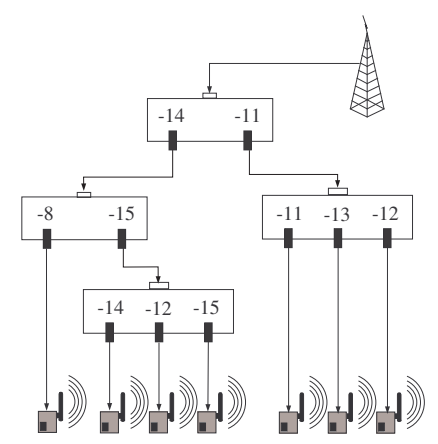

(b) A solution with $14 \mathrm{dBm}$ maximum power deviation.

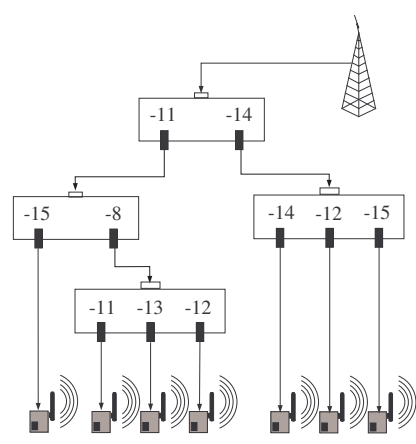

(c) Optimum with $4 \mathrm{dBm}$ maximum power deviation.

Figure 2: An illustrative example of equipment selection with respect to maximum power deviation. (BS power: $0 \mathrm{dBm}$; antenna power target: $-30 \mathrm{dBm}$ )

deviation among all the antennas. In Section 3, we formalize further the problem by means of mathematical notation.

Figure 2 provides an illustrative example of the equipment selection problem with four inner nodes and eight antennas. The number of ports of the inner nodes is either two or three. The BS output power is $0 \mathrm{dBm}$, and the power target equals $-30 \mathrm{dBm}$ for all antennas. Equipment types and their port gains (in $\mathrm{dB}$ ) are shown in Figure 2(a). The values are negative as they represent attenuation. The amount of attenuation caused by cables is a constant for each antenna, and for this example the value is considered zero. For the performance objective of maximum deviation from the target powers, Figure 2(b) shows a sub-optimal solution; the maximum deviation is $14 \mathrm{dBm}$. The global optimum, of which the maximum deviation is only $4 \mathrm{dBm}$, is illustrated in Figure 2(c). The optimum differs from the solution in Figure 2(b) in the selection of equipment type at two nodes, and the port assignment of the other two nodes.

From the above illustration, equipment selection for P-DAS falls into the domain of combinatorial optimization, as both the selection of equipment at each inner node as well as the assignment of ports to the outgoing edges involve discrete choice, and the number of possible port assignments is exponential in the number of edges. For a combinatorial optimization problem, theoretical insights of tractability and approximability, as well as algorithmic notions derived from the theoretical results are key aspects for problem solution. These aspects form 
the core of our study. Specifically, We present the following contributions for the equipment selection problem with both objectives. First, we prove that the problem is weakly NP-hard, even if the topology is a path. Second, we develop exact pseudopolynomial-time algorithms. Our analysis of algorithm complexity identifies the node degree and the range of possible power levels as the crucial parameters in the time required for global optimality. Third, we derive additive fully polynomial-time approximation schemes (FPTAS), enabling approximate solutions with progressively improving quality. Numerical results are provided to illustrate algorithm performance. Finally, we extend our results to account for installation costs.

\section{Literature Review}

There has been a significant amount of work measuring the potential and benefits of IB-DAS. In [24], extensive signal propagation measurements have been conducted to serve as a basis for assessing IB-DAS capacity. For high-speed downlink packet access (HSDPA), measurements of IB-DAS performance are presented in [16] to provide guidelines for system deployment, showing that IB-DAS can provide better capacity and coverage in comparison to the use of pico-cells. Moreover, the study in [16] reveals that sufficient coverage, which is strongly related to antenna output power, is the key factor in planning IB-DAS with HSDPA. Measurementbased coverage and capacity analysis for IB-DAS with universal mobile telecommunication system (UMTS) is provided in [7]. In [4], the authors focus on the energy efficiency aspect of an IB-DAS architecture. The simulation results demonstrate that IB-DAS has superior energy efficiency over an all-wireless system combining macro-cells and femto-cells.

Performance engineering of IB-DAS has been studied in several papers. In [1], the authors study spectral efficiency in respect to the number of antennas, subject to interference between antenna elements at different floors. For a similar setting, joint signal processing with interference cancellation is investigated in [2]. A mathematical channel model for indoor propagation of high building is proposed in $[19,20]$. The model as well as system simulations have been used to analytically and numerically characterize the achievable rate of IB-DAS. For a given 
IB-DAS topology, algorithms for transmission scheduling and resource coordination among the antennas are presented in [23]. For a comprehensive treatment of system specification and common engineering practices of deploying IB-DAS, we refer to the textbook by Tolstrup [25].

For mathematical optimization of IB-DAS deployment, an integer programming formulation for determining the cabling and equipment is presented in [9], with the primary objective of reducing cable cost. Due to the nature of integer programming, the approach admits solutions for relatively small scenarios only. In [3], the authors formulate the optimization problem of optimally locating the antennas and setting their output powers (which form part of the input to the problem we study) for coverage and interference avoidance. A simulated annealing algorithm is proposed and evaluated by comparing the results to that of uniform power.

\section{Problem Definition}

We represent an IB-DAS by triplet $T=(r, V, A)$, where $(V, A)$ is a directed tree with root $r \in V$. We denote by $n=|V|$ the total number of nodes. Given $T$, let $N_{v}=\{w \in V:(v, w) \in$ $A\}$ be the set of children of node $v \in V, V_{L}=\left\{v \in V: N_{v}=\emptyset\right\}$ be the set of leaf nodes, and $V_{I}=V \backslash V_{L}$ be the inner, non-leaf nodes. In P-DAS, the BS is directly connected to a single inner node, thus we can omit the BS, and assume that the tree is rooted at that inner node. The effect of omitting the BS can be compensated by adapting the target powers at the leaves.

For each node $v \in V$, we denote by $T_{v}$ the subtree of $T$ rooted at $v$. The depth of a node $v \in V$ is its distance from the tree root, and is equal to the number of nodes in the (unique) path connecting $r$ to $v$ in $T$. The path is denoted by $M(r, v)$.

An equipment type is characterized by the number of output ports and by the power gain of each port. Throughout the paper, we assume that the power data, i.e., power values as well as power gains, are defined upon an appropriate logarithmic scale so as to make all coefficient integrals. Note that this is equivalent to assuming data rationality. For the sake of generality, we allow both negative (attenuation) and positive (amplification) power gains, 
although P-DAS power equipment types always have negative gains.

A power equipment can be installed at an inner node $v \in V_{I}$ only if the number of output ports equals the number of outgoing arcs from $v$. All equipment types compatible with $v \in V_{I}$ have thus $\left|N_{v}\right|$ output ports, which we index by the set $\Lambda_{v}=\left\{1, \ldots,\left|N_{v}\right|\right\}$. These equipment types differ in their power settings. We define a compatible equipment for $v \in V_{I}$ by a port-gain mapping $g: \Lambda_{v} \rightarrow \mathbb{Z}$, associating to each port $\lambda \in \Lambda_{v}$ a corresponding power gain $g(\lambda) \in \mathbb{Z}$. We assume for each node $v \in V_{I}$, the problem input contains a specific set $G_{v}$ of compatible equipments, each described by a port-gain mapping. While compatibility is typically only determined by the number of ports, defining a node-individual set of compatible equipments allows more generality in modeling. We denote by $m=\max _{v \in V_{I}}\left|G_{v}\right|$ the maximum number of compatible equipment types among all inner nodes.

A port assignment for a node $v \in V_{I}$ is a bijection $\pi: N_{v} \rightarrow \Lambda_{v}$, assigning to each child node $w \in N_{v}$, and hence to each $\operatorname{arc}(v, w) \in A$, a corresponding port index $\pi(w) \in \Lambda_{v}$. Therefore, an equipment solution is a tuple $s=\left(g_{v}, \pi_{v}\right)_{v \in V_{I}}$, specifying for each node $v \in V_{I}$ the chosen compatible equipment $g_{v} \in G_{v}$ and port assignment $\pi_{v}$. Thus, given an equipment solution $s=\left(g_{v}, \pi_{v}\right)_{v \in V_{I}}$, for each node $v \in V_{I}$ the gain of the port used by the connection to a child $w \in N_{v}$ is equal to $g_{v}\left(\pi_{v}(w)\right)$. For the given tree $T$, let $p_{v} \in \mathbb{Z}$ be the target power for each leaf node $v \in V_{L}$, and let $\bar{p}$ be the power of the BS. Moreover, let $g_{a} \in Z$ be the power gain for each arc $a \in A$, which in a P-DAS is negative due to signal loss over the cables.

Throughout the paper, the term output power of a node refers to the power level prior to the loss due to the node's equipment. Given an equipment solution $s=\left(g_{v}, \pi_{v}\right)_{v \in V_{I}}$, the output power $p_{v}^{s}$ at each $v \in V$ can be computed by subtracting the signal loss along $M(r, v)$ from the output power $p_{r}^{s}=\bar{p}$ at the root. Formally, this is obtained by setting $p_{w}^{s}=p_{v}^{s}+g_{v}\left(\pi_{v}(w)\right)+g_{a}$ for each arc $a=(v, w) \in A$, proceeding by non-decreasing depth. Given a leaf node $v \in V_{L}$, we refer to the difference $p_{v}-p_{v}^{s}$ between the target power for $v$ and its output power as the signed deviation for $v$ in $s$, and refer to the absolute value $\left|p_{v}-p_{v}^{s}\right|$ as the deviation for $v$ in $s$. For the subtree of a node $v \in V$, the maximum and total deviation induced by the solution $s$ 
are denoted respectively by $\Delta(s, v)$ and $\Sigma(s, v)$. The entities are formally defined below.

$$
\Delta(s, v)=\max _{w \in T_{v} \cap V_{L}}\left|p_{w}-p_{w}^{s}\right|, \text { and } \Sigma(s, v)=\sum_{w \in T_{v} \cap V_{L}}\left|p_{w}-p_{w}^{s}\right|
$$

The performance metrics we consider are the maximum power deviation $\Delta(s)=\Delta(s, r)$ and the total power deviation $\Sigma(s)=\Sigma(s, r)$. The two resulting minimization problems are denoted min-max and min-sum, respectively, and are formalized as follows.

Input: $\quad$ A rooted tree $T=(r, V, A)$, a compatible equipment set $G_{v}$ for each inner node $v \in V_{I}$, a target power $p_{v}$ for each leaf node $v \in V_{L}$.

Output: An equipment selection solution $s=\left(g_{v}, \pi_{v}\right)_{v \in V_{I}}$.

Goal: $\quad$ Minimize $\Delta(s)$ for min-max, and minimize $\Sigma(s)$ for min-sum.

Table 1: A summary of the notation.

\begin{tabular}{|l|l|}
\hline \multicolumn{1}{|c|}{ Notation } & \multicolumn{1}{c|}{ Description } \\
\hline$r$ & Tree root \\
\hline$V$ & Set of nodes \\
\hline$A$ & Set of arcs, each connecting a node to one of its children \\
\hline$T=(r, V, A)$ & Rooted tree representing an in-building P-DAS topology \\
\hline$n$ & Number of nodes, i.e., $n=|V|$ \\
\hline$V_{L}$ & Set of leaves (antennas) \\
\hline$V_{I}$ & Set of inner nodes $\left(V_{I}=V \backslash V_{L}\right)$ \\
\hline$N_{v}$ & Set of children of inner node $v \in V_{I}$ \\
\hline$M(r, v)$ & The unique path connecting $r$ to node $v$ \\
\hline $\bar{p}$ & Output power of the root \\
\hline$g_{a}$ & Power gain of arc $a$ \\
\hline$\Lambda_{v}$ & Set of port indices of node $v$, i.e., $\Lambda_{v}=\left\{1, \ldots,\left|N_{v}\right|\right\}$ \\
\hline$g: \Lambda_{v} \rightarrow \mathbb{Z}$ & Port-gain mapping representing a compatible equipment for $v \in V_{I}$ \\
\hline$g(\lambda)$ & The port gain given by $g$ for port $\lambda$ \\
\hline$G_{v}$ & $\begin{array}{l}\text { Set of compatible equipments for inner node } v, \text { each represented by } \\
\text { a port-gain mapping }\end{array}$ \\
\hline$m$ & $\begin{array}{l}\text { Maximum number of compatible equipments over all inner } \\
\text { nodes, i.e., } m=\text { max } \\
v \in V_{I}\left|G_{v}\right|\end{array}$ \\
\hline$\pi: N_{v} \rightarrow \Lambda_{v}$ & Bijection that maps the children of inner node $v$ to its ports \\
\hline$s=\left(g_{v}, \pi_{v}\right)_{v \in V_{I}}$ & A complete solution of equipment selection \\
\hline
\end{tabular}

Table 1 provides a summary of the notation introduced in this section. For the sake of simplicity, in the remainder of the paper we assume $\bar{p}=0$ and $g_{a}=0$ for all $a \in A$. The 
assumptions do not cause any loss of generality, because the original values can be accounted for by simply shifting the target powers.

\section{Problem Complexity}

Before considering solution algorithms, we formalize the complexity of the equipment selection problem for both objectives. The results and proofs are provided below.

Lemma 1. Both min-max and min-sum are weakly NP-hard, even if $T$ is a path.

Proof. The proof uses a reduction from the SUBSET-SUM problem [10]. In SUBSET-SUM, we are given $h$ integers $q_{1}, \ldots, q_{h} \in \mathbb{N}$ and an integer $b \in \mathbb{N}$. The problem asks for a binary vector $x \in\{0,1\}^{h}$ for which $\sum_{i=1}^{h} q_{i} x_{i}=b$. Given an instance of SUBSET-SUM, consider $T$ of $h+2$ nodes $r, 1,2, \ldots, h+1$, and $h+1$ arcs forming a path from $r$ to $h+1$. The leaf node $h+1$ is an antenna. As nodes $1, \ldots, h$ have only one outgoing arc, it suffices to specify a compatible equipment type by one power coefficient. For each of the arcs in the sequence $(1,2),(2,3), \ldots,(h, h+1)$, there are two compatible equipment types with one output port. The power coefficients are 0 and $q_{v}$ for arc $(v, v+1), v=1, \ldots, h$. Setting the target power at the node $h+1$ for both min-max and min-sum to $b$, there is a solution with zero deviation if and only if the SUBSET-SUM instance is satisfiable, and the theorem follows.

In the proof above, the candidate equipment type is constrained to be node-specific. That is, the equipment with coefficient $q_{v}$ is eligible at an inner node $v$ but not for an inner node $w \neq v$, although both have one outgoing arc. In the following, we prove that the complexity conclusion remains without this restriction.

Theorem 1. Both min-max and min-sum are weakly NP-hard, even if all inner nodes have the same set of compatible equipment types.

Proof. To prove this, we use a reduction from the integer version of SUBSET-SUM (ISS).

The problem asks for an integer vector $x \in \mathbb{N}^{h}$ for which $\sum_{i=1}^{h} q_{i} x_{i}=b$. We can assume 
without loss of generality that no integer $q_{i}$ is divisible with any other integer $q_{j}$ with $j \neq i$, as otherwise $q_{j}$ could be removed. Given an instance of ISS, we define $\gamma_{i}=\left\lfloor\log _{2} \frac{b}{q_{i}}\right\rfloor$ and denote by $\Gamma_{i}$ the set of integers going between 0 and $\gamma_{i}$ for each $i \in H$ where $H=\{1, \ldots, h\}$. We then construct a path $T$ as follows. There are $2+h+\sum_{i \in H} \gamma_{i}$ nodes. The first node is root $r$. For each $i \in H$ and $j \in \Gamma_{i}$, nodes $v_{i, 0}, v_{i, 1}, \ldots, v_{i, \gamma_{i}}$ are defined. Finally, there is a sink node (i.e., the antenna), denoted by $v_{h+1,0}$. The path starts at $r$ that has a single arc to $v_{1,0}$. For each $i \in H$ and $j \in \Gamma_{i}$, we add $\operatorname{arc}\left(v_{i, j}, v_{i, j+1}\right)$ if $j<\gamma_{i}$, otherwise $\left(v_{i, j}, v_{i+1,0}\right)$ is added. The resulting graph is a directed path leading the root $r$ to the sink $v_{h+1,0}$. We set the target power of the sink to $b$. The set of compatible equipment types for all inner nodes has $\sum_{i \in H} \gamma_{i}+1$ elements. The equipment types all have one output port but differ in their power gains. Specifically, the set of power gains is $\left\{2^{j} q_{i}: i \in H, j \in \Gamma_{i}\right\} \cup\{0\}$.

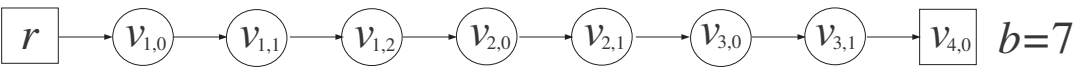

Set of power gains: $\left\{2^{0} \cdot 1,2^{1} \cdot 1,2^{2} \cdot 1,2^{0} \cdot 2,2^{1} \cdot 2,2^{0} \cdot 3,2^{1} \cdot 3,0\right\}$

Figure 3: An illustration of the reduction.

We provide an illustrative example of path $T$ used in the proof in Figure 3. Consider the ISS instance $x_{1}+2 x_{2}+3 x_{3}=7$, for which $\gamma_{i}=\left\lfloor\log _{2} \frac{b}{q_{i}}\right\rfloor=2,1,1$, for $i=1,2$, and 3 , respectively. Hence the sets $\Gamma_{1}=\{0,1,2\}, \Gamma_{2}=\{0,1\}$, and $\Gamma_{3}=\{0,1\}$, and thus the path has nine nodes in total. The inner nodes consist in three segments with 3,2 , and 2 nodes, respectively, corresponding to the three items in the ISS instance. All inner nodes share a common pool of single-port equipment types, of which the gain options form the set $\left\{2^{j} q_{i}, j \in \Gamma_{i}, i=1, \ldots, 3\right\}$. Finally, the target power value is the right-hand side of the ISS instance.

Given a feasible ISS solution $x$, there is a solution $s=\left(g_{v}\right)_{v \in V_{I}}$ with zero deviation, by setting $g_{v}(1)=2^{j} q_{i}$ if the $j$-th digit of the binary encoding of $x_{i}$ is non-zero, and $g_{v}(1)=0$ otherwise, for each inner node $v=v_{i, j}, i \in H, j \in \Gamma_{i}$. Conversely, assume that $s=\left(g_{v}\right)_{v \in V_{I}}$ is a solution with zero deviation, and let $V_{i}(s)$ be the set of nodes for which $g_{v}=2^{j} q_{i}$ for $v=$ 
$v_{i, j}, i \in H, j \in \Gamma_{i}$. A feasible ISS solution $x$ is obtained by setting $x_{i}=\sum_{v \in V_{i}(s)} g_{v} / q_{i}, i \in H$. Hence there is a one-to-one correspondence between feasible solutions of ISS and equipment selection with zero deviation, and the theorem follows.

In the remainder of this paper we develop pseudopolynomial-time and exact algorithms (Section 5), as well as polynomial-time and approximation schemes (Section 6). Moreover, a greedy algorithm is considered in Section 7. Because the optimum of min-max and min-sum can attain value zero, no multiplicative approximation factor can be guaranteed in general. Our goal of solution approximation is to derive algorithms with an additive approximation factor, or a combination of additive and multiplicative factors. To express pseudopolynomiality, we define the following lower and upper bounds on the value of signed deviation:

$$
\begin{aligned}
& \bar{l}=\min _{v \in V_{L}}\left(p_{v}-\sum_{w \in M(r, v)} \max \left\{0, \max _{g \in G_{w}} \max _{\lambda \in \Lambda_{w}} g(\lambda)\right\},\right. \\
& \bar{u}=\max _{v \in V_{L}}\left(p_{v}-\sum_{w \in M(r, v)} \min \left\{0, \min _{g \in G_{w}} \min _{\lambda \in \Lambda_{w}} g(\lambda)\right\}\right) .
\end{aligned}
$$

Note that the values also set the range of possible power levels of the nodes in any solution to min-max and min-sum. In addition, the bounds apply to the partial solutions defined on a subtree $T_{v}$ in which $v$ has zero input power. We define $\Omega=\{\bar{l}, \ldots, \bar{u}\}$, which is the set of integers within the range defined by the two bounds, and $U=|\Omega|$. Note that $U$ is bounded by a polynomial in $n, m$, and the power data.

\section{Pseudopolynomial-time Algorithms}

The key notion of our algorithmic design is labeling. We keep for each node $v \in V_{I}$ a set $L_{v}$ of unique labels. A label $\ell \in L_{v}$ represents a partial equipment selection solution for the subtree $T_{v}$, treating $v$ as the root. For min-max, a label $\ell \in L_{v}$ is a pair of integers $(l, u)$, with $l \leq u$. For min-sum, a label $\ell \in L_{v}$ is a function $\ell: \Omega \rightarrow \mathbb{N}$, with the notational convention that 
$\ell(x)=0$ if $x \notin \Omega$. In the sequel, we use $f(\ell, p)$ to denote the objective function value, i.e., maximum or total power deviation, induced by label $\ell \in L_{v}$, assuming that $v$ has power $p$.

Property 1 (min-max). If the power of the node $v$ equals $p$, for any $p \in \Omega$, then every label $\ell=(l, u) \in L_{v}$ corresponds to a partial solution for $T_{v}$ for which all signed deviations are between $l-p$ and $u-p$ with both extremes attained, giving maximum deviation $f(\ell, p)=$ $\max \{|l-p|,|u-p|\}$ for the subtree $T_{v}$.

Property 1 (min-sum). If the power of the node $v$ equals $p$, for any $p \in \Omega$, then every label $\ell \in L_{v}$ with $\ell: \Omega \rightarrow \mathbb{N}$ corresponds to a partial solution for $T_{v}$ for which there are exactly $\ell(d)$ leaves in $T_{v}$ with signed deviation $d-p$, giving a total deviation of $f(\ell, p)=\sum_{d \in \Omega}|d-p| \ell(d)$ for the subtree $T_{v}$.

For min-max, one has $\bar{l} \leq l \leq u \leq \bar{u}$ for any label $\ell=(l, u)$, so that $\ell \in \Omega \times \Omega$. For min-sum, a simple way of representing a label $\ell: \Omega \rightarrow \mathbb{N}$ is a vector of $U$ entries, one for each value in $\Omega$. One can however observe that for any label $\ell \in L_{v}$ of a node $v, \sum_{d \in \Omega} \ell(d)$ is precisely the number of leaves in $T_{v}$. Therefore, labels can have a compact representation in form of a list of pairs $(d, \ell(d))$, one for each $d \in \Omega$ for which $\ell(d)>0$. This observation allows for improving the running time, but for the sake of simplicity and clarity it is not taken into account in the algorithm analysis.

We proceed with two steps of algorithmic development. We first consider a basic algorithm, given in Algorithm 1, that runs in pseudopolynomial time for trees with bounded degree. Then we derive an improved algorithm of pseudopolynomial time complexity for arbitrary instances.

Algorithm 1 proceeds as follows. For each leaf $v \in V_{L}$ it creates a single label $\delta_{v}$. This label is set to $\delta_{v}=\left(p_{v}, p_{v}\right)$ for min-max, and to the indicator function $\delta_{v}: \Omega \rightarrow \mathbb{N}$, defined by $\delta_{v}(d)=1$ if $d=p_{v}$ and $\delta_{v}(d)=0$ otherwise, for min-sum. In both cases, the rationale is that power level $p$ at a leaf $v \in V_{L}$ results in $p_{v}-p$ as the signed deviation from target $p_{v}$, and hence the definition of label $\delta_{v}$ gives $f\left(\delta_{v}, p_{v}\right)=0$ for both min-max and min-sum. The algorithm then processes the inner nodes in a bottom-up order, that is, by non-decreasing depth (with arbitrary tie resolution). For each inner node, two steps are performed. First, the 

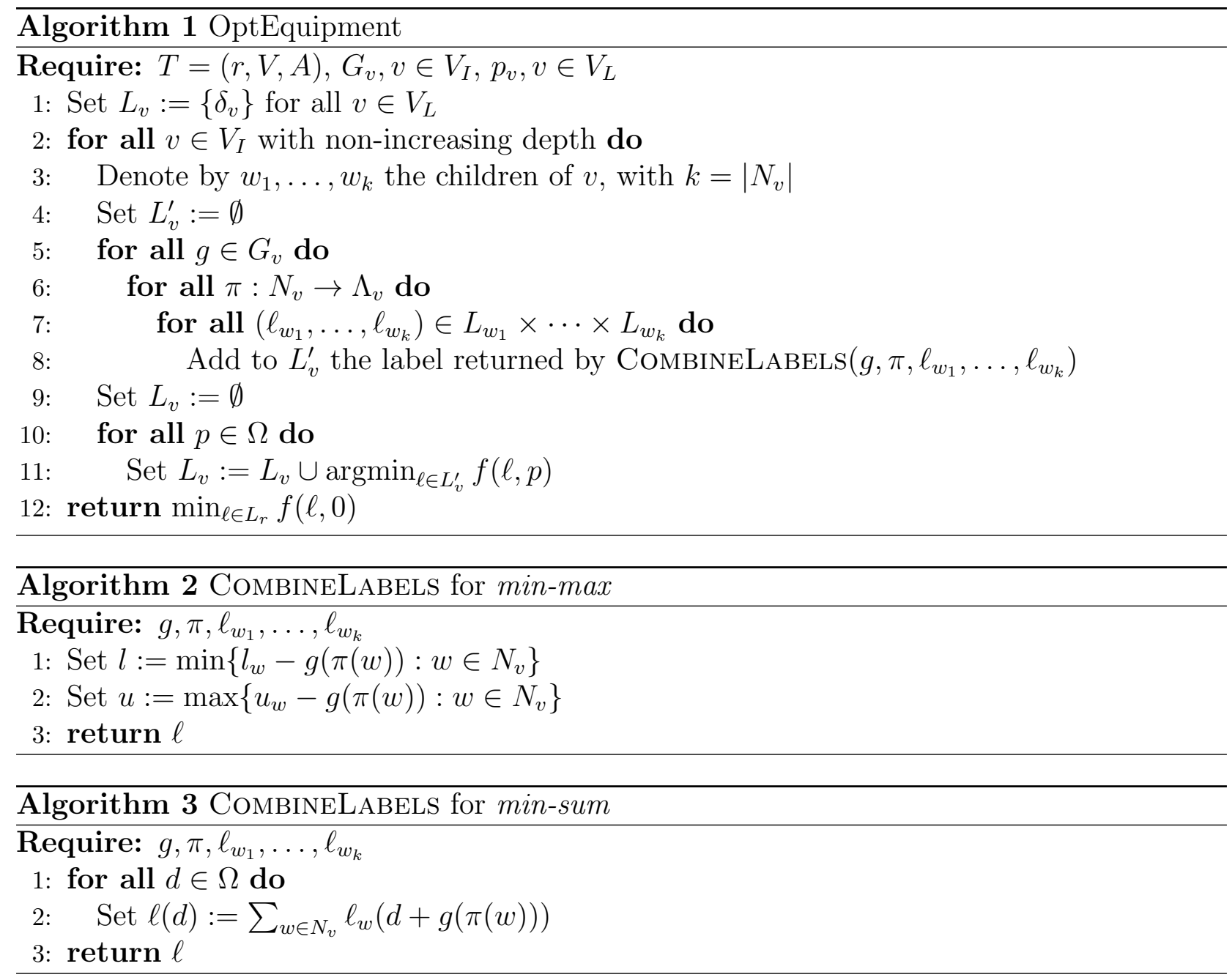

algorithm accumulates labels in a set $L_{v}^{\prime}$, creating a label for each combination of compatible equipment, port assignment, and choice of label for each child. Label generation is performed through CombineLabels, which is described in Algorithm 2 for min-max and in Algorithm 3 for min-sum. In both cases, the shifting by $\pm g(\pi(w))$ is due to the fact that the algorithm proceeds in a bottom-up fashion. Next, the algorithm generates label set $L_{v}$ by choosing from $L_{v}^{\prime}$ a label for each possible value of output power at $v$. Namely, for each $p \in \Omega$, the algorithm adds to $L_{v}$ a label $\ell \in L_{v}^{\prime}$ that minimizes $f(\ell, p)$. The key idea is that, if Property 1 is satisfied, this label corresponds to an optimal solution for the subtree $T_{v}$ if $v$ attains power $p$. The root node is the last node processed. As the root node has zero output power, the algorithm finally returns the value of a label $\ell \in L_{v}$ that minimizes $f(\ell, 0)$, which is an optimal solution. In the following, we prove that the labels generated by Algorithm 1 indeed respect Property 1. 
Lemma 2. In Algorithm 1, Property 1 holds true for each node $v \in V$ and each label $\ell \in L_{v}$.

Proof. We use induction for the proof. Property 1 clearly holds true for the label $\delta_{v}$ of each leaf node $v \in V_{L}$. Consider an inner node $v \in V_{I}$ and a label $\ell \in L_{v}$, and assume by induction that Property 1 is satisfied for each child $w \in N_{v}$ and each label in $L_{w}$. As $L_{v}$ contains $\ell$, there exists a compatible equipment $g \in G_{v}$, a port assignment $\pi: N_{v} \rightarrow \Lambda_{v}$, and a label $\ell_{w} \in L_{w}$ for each child $w \in N_{v}$ for which $\ell=\operatorname{CombineLabels}\left(g, \pi, \ell_{w_{1}}, \ldots, \ell_{w_{k}}\right)$. By the induction hypothesis, for any $w \in N_{v}$ there exists a partial solution $s_{w}$ for $T_{w}$ that respects Property 1 for label $\ell_{w}$. Consider solution $s$ for $T_{v}$, obtained by combining $s_{w}$ for each $w \in N_{v}$ with the assignment $(g, \pi)$ for $v$. Let $p_{u}^{s}$ be the output power at $u \in T_{v}$ in solution $s$, defined assuming output power $p$ at $v$. Note that $p_{w}^{s}=p+g(\pi(w))$ for each child $w \in N_{v}$.

Consider min-max, and let $\ell=(l, u)$. For each solution $s_{w}$, Property 1 guarantees by the induction hypothesis that all signed deviations in $s_{w}$ are within $l_{w}-p_{w}^{s}=l_{w}-p-g(\pi(w))$ and $u_{w}-p_{w}^{s}=u_{w}-p-g(\pi(w))$, with both extremes attained. By the construction of $l$ and $u$ in CombineLabels, for each $w \in N_{v}$ one has $l \leq l_{w}-g(\pi(w))$ and $u \geq u_{w}-g(\pi(w))$, which implies $l-p \leq l_{w}-p_{w}^{s}$ and $u-p \geq u_{w}-p_{w}^{s}$. This allows to conclude that all signed deviations in the solution $s$ are within $l-p$ and $u-p$. Moreover, as $l=l_{w^{\prime}}-g\left(\pi\left(w^{\prime}\right)\right)$ and $u=u_{w^{\prime \prime}}-g\left(\pi\left(w^{\prime \prime}\right)\right)$ for some $w^{\prime}, w^{\prime \prime} \in N_{v}$, the signed deviations $l_{w^{\prime}}-p_{w^{\prime}}^{s}=l-p$ and $u_{w^{\prime \prime}}-p_{w^{\prime \prime}}^{s}=u-p$ are both present in $s$.

Consider next min-sum. For each solution $s_{w}$, Property 1 guarantees by the induction hypothesis that exactly $\ell_{w}(d+g(\pi(w)))$ leaf nodes in $T_{w}$ have signed deviation $d+g(\pi(w))-$ $p_{w}^{s}=d-p$. Therefore, in the solution $s$ there will be in total $\ell(d)=\sum_{w \in N_{v}} \ell_{w}(d+g(\pi(w)))$ leaves with signed deviation $d-p$.

Using Lemma 2, we are able to prove the optimality of Algorithm 1. The result, along with the running time of the algorithm, is formalized hereafter.

Theorem 2. Algorithm 1 returns in time $O\left(n\left(m k ! U^{k} c_{1}+U^{2} c_{2}\right)\right)$ the optimum of the equipment selection problem, where $k=\max _{v \in V_{I}}\left|N_{v}\right|$ is the maximum node out-degree, $c_{1}$ is the running 
time for a call to COMBINELABELS and $c_{2}$ is the time for evaluating $f(\ell, p)$ for a label $\ell$ and a value $p \in \Omega$.

Proof. The running time analysis is straightforward by inspecting the Algorithm 1, and noting that there are at most $k$ ! possible port assignments, and that $\left|L_{v}\right| \leq U$ for every node $v$.

Let $A L G$ be the value returned by Algorithm 1. By Lemma 2, there exists a solution with objective value $A L G$, obtained by a trivial modification of Algorithm 1. To prove optimality, assume by contradiction that there exists a solution $s=\left(g_{v}, \pi_{v}\right)_{v \in V_{I}}$ with objective value $f\left(\ell_{r}^{s}, 0\right)$ such that $f\left(\ell_{r}^{s}, 0\right)<A L G$. Solution $s$ induces a label $\ell_{v}^{s}$ for each $v \in V$, defined recursively by setting $\ell_{v}^{s}=\delta_{v}$ for $v \in V_{L}$, and setting $\ell_{v}^{s}$ to the label returned by CombineLABELS when called with compatible equipment $g_{v}$, port assignment $\pi_{v}$, and label $\ell_{w}^{s}$ for each $w \in N_{v}$.

As $f\left(\ell_{r}^{s}, 0\right)<A L G$, clearly $\ell_{r}^{s} \notin L_{r}$. As $\ell_{v}^{s} \in L_{v}$ for all $v \in V_{L}$, there must be a node $v \in V_{I}$ for which $\ell_{w}^{s} \in L_{w}$ for all children $w \in N_{v}$, but $\ell_{v}^{s} \notin L_{v}$. Because $\ell_{w}^{s} \in L_{w}$ for all children $w \in N_{v}, \ell_{v}^{s} \in L_{v}^{\prime}$ by construction, since Algorithm 1 tries all possible compatible equipments, port assignments, and children labels. Thus, if $\ell_{v}^{s} \notin L_{v}$ is assumed, there must be some label $\tilde{\ell} \in L_{v}$ for which $f\left(\tilde{\ell}, p_{v}^{s}\right) \leq f\left(\ell_{v}^{s}, p_{v}^{s}\right)$. Consider the partial solution $\tilde{s}_{v}$ corresponding to the label $\tilde{\ell}$ for the subtree $T_{v}$. The existence of such a solution is guaranteed by Property 1 and Lemma 2. Then, we construct a new solution $\tilde{s}$, which is equal to $\tilde{s}_{v}$ in $T_{v}$, and equal to $s$ elsewhere. Note that $\tilde{\ell}=\ell_{v}^{\tilde{s}_{v}}=\ell_{v}^{\tilde{s}}$. Because $f\left(\tilde{\ell}, p_{v}^{s}\right) \leq f\left(\ell_{v}^{s}, p_{v}^{s}\right)$, and the output power of all nodes outside $T_{v}$ is the same in $s$ and $\tilde{s}$, by the label characterization in Property 1 it is easy to see that $f\left(\ell_{r}^{\tilde{s}}, 0\right) \leq f\left(\ell_{r}^{s}, 0\right)$. Moreover, we now have $\ell_{v}^{\tilde{s}} \in L_{v}$, and the only labels for which this property may be lost are those corresponding to nodes preceding $v$ in the path $M(r, v)$. Repeating the procedure, we eventually obtain a solution $\hat{s}$ for which $f\left(\ell_{r}^{\hat{s}}, 0\right) \leq f\left(\ell_{r}^{s}, 0\right)$ and $\ell_{r}^{\hat{s}} \in L_{r}$, contradicting the assumption $f\left(\ell_{r}^{s}, 0\right)<A L G$, and the theorem follows.

By Theorem 2, Algorithm 1 solves min-max and min-sum in pseudopolynomial time for any instance with bounded out-degree. Our next algorithm, of which a formal description is given in Algorithm 4, extends the labeling idea for solving arbitrary instances in pseudopolynomial time. The idea is to directly generate optimal labels. More precisely, for each node 
$v \in V_{I}$, Algorithm 4 considers all combinations of potential output power values $p \in \Omega$ and compatible equipment types $g \in G_{v}$. For each combination of $p$ and $g$, the algorithm generates a single label through a routine called OptLABEL. This routine finds a port assignment $\pi$ and a label selection $\left(\ell_{w_{1}}, \ldots, \ell_{w_{k}}\right) \in L_{w_{1}} \times \cdots \times L_{w_{k}}$ for which $f(\ell, p)$ is minimized, for $\ell=$ CombineLabels $\left(g, \pi, \ell_{w_{1}}, \ldots, \ell_{w_{k}}\right)$. Therefore, if $g$ is installed at $v$ and $v$ has output power $p$, this label represents an optimal solution for the subtree $T_{v}$. For each output power $p \in \Omega$, the best label among all possible equipment types $g \in G_{v}$ is then added to $L_{v}$.

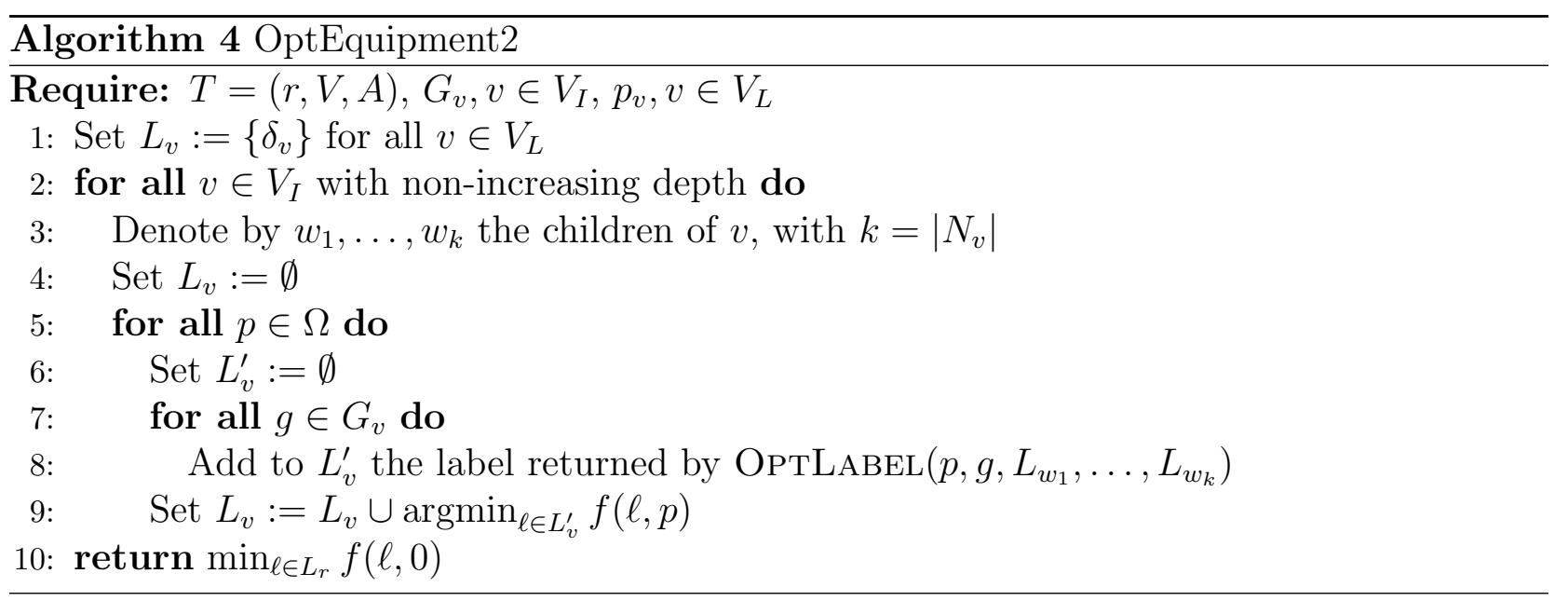

As for Combinelabels, OptLabel is different for min-max and min-sum. For the former, it requires solving a bottleneck linear assignment problem, while for the latter it involves the solution of a linear assignment problem. Formally, we construct a bipartite graph with node set composed of the output ports $\Lambda_{v}$ on one side and the child nodes $N_{v}$ on the other. For each $\lambda \in \Lambda_{v}$ and $w \in N_{v}$, we add an edge $(\lambda, w)$ with weight $\min _{\ell \in L_{w}} f(\ell, p+g(\lambda))$. This edge weight corresponds to the optimal objective function contribution from the leaves in $T_{w}$ if node $v$ attains power level $p$ and the edge $(v, w)$ gets assigned to port $\lambda$.

An example of the graph construction for min-max is given in Figure 4, for a node $v$ with given equipment (in form of a port-gain mapping) $g \in G_{v}$ under consideration. Node $v$ has two ports. The child nodes are $w_{1}$ and $w_{2}$, each having two labels. In the constructed bipartite graph, the two ports are represented by two separate nodes (indexed one and two in the illustration), each having its edges to $w_{1}$ and $w_{2}$. The edge weights are set to $\min _{\ell \in L_{w}} f(\ell, p+$ 


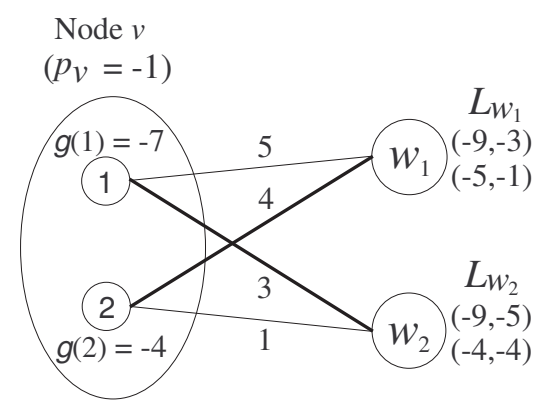

Figure 4: An illustration of the graph construction for OptLABEL in Algorithm 4.

$g(\lambda)$ ), with $\lambda \in\{1,2\}$ and $w \in\left\{w_{1}, w_{2}\right\}$. For instance, for $i=1$ and $w=w_{1}$, the first label leads to $\max \{|-9-(-1-7)|,|-3-(-1-7)|\}=5$, and the second label leads to $\max \{|-5-(-1-7)|,|-1-(-1-7)|\}=7$ (see the definition of label of min-max at the beginning of this section and Property 1). Hence the edge weight is set to $\min \{5,7\}=5$. Other edge weights are obtained following the same line of computation. In the figure, the optimum for the bottleneck assignment problem for min-max is represented by the thick edges. For min-sum, the structure of this bipartite graph remains, with the only difference that the weights are set based on total power deviation (see Property 1 for min-sum).

Given the bipartite graph, the assignments minimizing the maximum weight and total weight are optimal for min-max and min-sum, respectively The former can be solved in time $O\left(k^{2.5} \log k\right)$ by binary search (see e.g. [8] and the references therein) and the Hopcroft-Karp algorithm [14]. The latter can be solved in time $O\left(k^{3}\right)$ with the Jonker-Volgenant algorithm for the linear assignment problem [18]. The correctness and complexity of Algorithm 4 is a direct consequence of the correctness of Algorithm 1, considering that the set of labels $L_{v}$ generated by both algorithms has the same property. We can thus state the following result.

Theorem 3. Algorithm 4 returns in time $O(n m U c)$ the optimum of the equipment selection problem, where $c$ is the running time of OPTLABEL.

Both Algorithm 1 and Algorithm 4 can be viewed as dynamic programming schemes in trees. As was shown in [17], such schemes sometimes enjoy improved running times by replacing the bottom-up traversal with a so-called left-right traversal. Since in our problem the 
optimal value at a node depends on an optimal bijective assignment of ports to child nodes, it seems difficult to apply such techniques here.

\section{Additive approximation schemes}

An additive polynomial-time approximation scheme (PTAS) is a type of approximation algorithm that, for each desired precision $\epsilon$, provides a solution of value at most $O P T+\epsilon U B D$, where $O P T$ denotes the optimal value and $U B D$ is some input-dependent upper bound, with a running time that is polynomial in the input size. If the running time is also polynomial in $\frac{1}{\epsilon}$, then the algorithm is an additive fully-polynomial-time approximation scheme (FPTAS).

We develop additive approximation schemes for min-max and min-sum based on the pseudopolynomial-time algorithms and notion of discretization. Given the desired precision $\epsilon$, the idea is to appropriately scale the target powers and port gains, such that the algorithms in Section 5 have a running time that is polynomial in the size of the modified input and $\frac{1}{\epsilon}$. Specifically, for a discretization step $\sigma \in \mathbb{N}$, of which the value will be made precise later, we define a modified input of min-max and min-sum by rounding all target powers $p_{v}, v \in V_{L}$, as well as all port gains $g(\lambda), \lambda \in \Lambda_{v}, g \in G_{v}, v \in V_{I}$, to the closest multiple of $\sigma$. Hereafter, we denote by $\tilde{p}_{v}, v \in V_{L}$ and $\tilde{g}(\lambda), \lambda \in \Lambda_{v}, g \in G_{v}, v \in V_{I}$, the discretized coefficients. We show that running the algorithms in Section 5 with the modified input leads to an FPTAS.

Theorem 4. The equipment selection problem admits an additive FPTAS.

Proof. We show that applying Algorithms 1 or 4 to an appropriately discretized input yields a solution performing no worse than $O P T+\epsilon U$. For the solution $s$ and the node $v$, let $p_{v}^{s}$ and $\tilde{p}_{v}^{s}$ be the output powers calculated with respect to the original and new coefficients, respectively. By the rounding operation, for each leaf $w \in V_{L}$ we have $\left|p_{w}-\tilde{p}_{w}\right| \leq \frac{\sigma}{2}$. Moreover, for each node $v \in V$ the absolute difference $\left|p_{v}^{s}-\tilde{p}_{v}^{s}\right|$ is at most $\frac{\sigma}{2}$ times the length of the path $M(r, v)$, which gives the worst case bound $\left|p_{v}^{s}-\tilde{p}_{v}^{s}\right| \leq \frac{n-1}{2} \sigma$. The observations imply that for each leaf node $w \in V_{L}$ we have $\left|p_{w}-\tilde{p}_{w}\right|+\left|p_{w}^{s}-\tilde{p}_{w}^{s}\right| \leq \frac{n}{2} \sigma$, which allows to conclude that 
$\left|\tilde{p}_{w}-\tilde{p}_{w}^{s}\right| \leq\left|p_{w}-p_{w}^{s}\right|+\frac{n}{2} \sigma$ as well as $\left|p_{w}-p_{w}^{s}\right| \leq\left|\tilde{p}_{w}-\tilde{p}_{w}^{s}\right|+\frac{n}{2} \sigma$. We denote by $\bar{s}$ the solution returned by the algorithm with the discretized input, and by $s^{*}$ an optimal solution. We also denote by $A L G$ and $O P T$ the objective values of $\bar{s}$ and $s^{*}$, respectively, both evaluated with

respect to the original input. That is, $A L G=\max _{v \in V_{L}}\left|p_{v}-p_{v}^{\bar{s}}\right|$ and $A L G=\sum_{v \in V_{L}}\left|p_{v}-p_{v}^{\bar{s}}\right|$ for min-max and min-sum, respectively, for which the following results hold.

$$
\begin{aligned}
& \max _{v \in V_{L}}\left|p_{v}-p_{v}^{\bar{s}}\right| \leq \frac{n}{2} \sigma+\max _{v \in V_{L}}\left|\tilde{p}_{v}-\tilde{p}_{v}^{\bar{s}}\right| \leq \frac{n}{2} \sigma+\max _{v \in V_{L}}\left|\tilde{p}_{v}-\tilde{p}_{v}^{s^{*}}\right| \leq n \sigma+\max _{v \in V_{L}}\left|p_{v}-p_{v}^{s^{*}}\right|=O P T+n \sigma \\
& \sum_{v \in V_{L}}\left|p_{v}-p_{v}^{\bar{s}}\right| \leq \frac{n^{2}}{2} \sigma+\sum_{v \in V_{L}}\left|\tilde{p}_{v}-\tilde{p}_{v}^{\bar{s}}\right| \leq \frac{n^{2}}{2} \sigma+\sum_{v \in V_{L}}\left|\tilde{p}_{v}-\tilde{p}_{v}^{\bar{s}^{*}}\right| \leq n^{2} \sigma+\sum_{v \in V_{L}}\left|p_{v}-p_{v}^{\bar{s}^{*}}\right|=O P T+n^{2} \sigma .
\end{aligned}
$$

By setting $\sigma=\left\lceil\frac{\epsilon U}{n}\right\rceil$ for min-max and $\sigma=\left\lceil\frac{\epsilon U}{n^{2}}\right\rceil$ for min-sum, we obtain $A L G \leq O P T+\epsilon U$. To quantify the running time, it is sufficient to consider $O(U / \sigma)$ out of the $U$ values in $\Omega$, because all power coefficients in the discretized input are multiples of $\sigma$. This yields a running time of $O\left(n\left(m k ! q^{k} c_{1}+q^{2} c_{2}\right)\right)$ for Algorithm 1, and $O(n m q c)$ for Algorithm 4, where $q=\frac{n}{\epsilon}$ for $\min$-max and $q=\frac{n^{2}}{\epsilon}$ for min-sum, $k=\max _{v \in V_{I}}\left|N_{v}\right|$ is the maximum out-degree, and $c_{1}, c_{2}$ and $c$ are defined as in Theorems 2-3. In conclusion, Algorithm 1 yields an additive FPTAS for fixed $k$, while Algorithm 4 yields an additive FPTAS for the general case.

\section{Greedy Solution}

For the purpose of comparison, we also consider a greedy approach for min-sum and min-max. The principle of greedy algorithms has been widely adopted for NP-hard problems. Similar to the previous algorithms, the greedy algorithm here makes equipment selection following the bottom-up order of the inner nodes of tree $T$. For each inner node $v \in V_{I}$, the greedy algorithm considers the set of compatible equipment types $G_{v}$, and, for each type, all its port assignments. For each of such selections, the power deviations of the leaf nodes in subtree $T_{v}$ are calculated by taking the BS power, and adding the port gains of the current inner node as well as all those of the downstream inner nodes in subtree $T_{v}$. The equipment type and port 
assignment yielding the smallest sum and maximum power deviations for $T_{v}$ are then selected for min-sum and min-max, respectively. The algorithm terminates when it has processed all the inner nodes. The procedure is formalized in Algorithm 5.

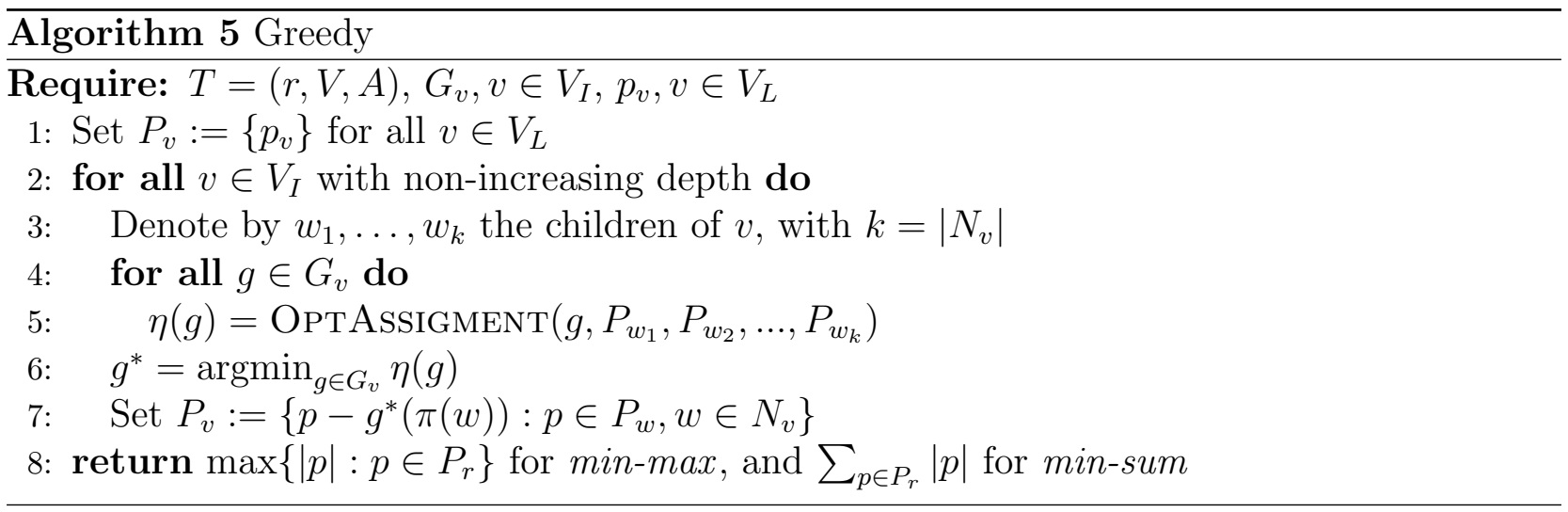

The computations in OptAssigment are similar to those in OPTLABEL. The difference lies only in the way of computing the edge weight. For $\min -\max$, the weight is $\max _{p \in P_{w}} \mid p-$ $g(\pi(w)) \mid$ whereas for min-sum it is $\sum_{p \in P_{w}}|p-g(\pi(w))|$.

\section{Numerical Illustration}

In this section we provide numerical results. The number of nodes of the tree scenarios ranges between 20 and 400. The maximum out-degree $k=5$. The number of children of each inner node follows a uniform and random distribution within $[2,5]$. Following the description of P-DAS in $[9,15]$, the root power is set to $50 \mathrm{dBm}$, and the antenna target power is an integer in range $[0,10] \mathrm{dBm}$. As was mentioned earlier, the power of the root in the algorithmic computations can be set to be zero, with the target powers shifted accordingly. For each of the four possible values of inner node degree in range $[2,5]$, there are five equipment types that differ in the port gains. The port gains are in the interval $[-15,0] \mathrm{dB}$ and integral. All computations have been conducted on a computer equipped with a dual core AMD 2.71 GHz CPU and 1.75 GB RAM. For each network size, the algorithms have been applied to 10 instances and the average values are used for performance comparison. 


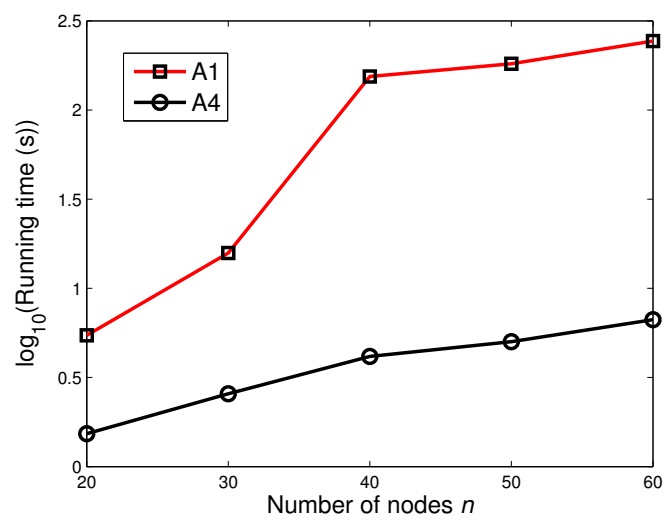

Figure 5: A comparison between Algorithm 1 and Algorithm 4 for min-max.

Figure 5 displays the computing time (in logarithmic scale) of the two exact algorithms for min-max. In this figure, the $y$-axis is in logarithmic scale for better visibility. From the figure, it is apparent that Algorithm 1 is inferior in comparison to Algorithm 4, by at least one order of magnitude, as the former explicitly enumerates the possible port assignments. In addition, the time difference grows rapidly in network size $n$. For min-sum, the difference becomes even larger and Algorithm 1 is not able to tackle instances with $n>20$ within any reasonable time, due to which we omit detailed comparison for min-sum. All these observations are fully in line with the complexity analysis in Section 5.

Based on the above results, in the following we focus on the additive FPTAS of Algorithm 4. For the FPTAS, note that the discretization step $\sigma$ is a positive integer. Given $\sigma$, there is a corresponding maximum $\epsilon$, for which the gap $\epsilon U$ is however instance-dependent. Moreover, as the power and gain values are integral (in $\mathrm{dBm}$ and $\mathrm{dB}$, respectively), setting $\sigma=1$ is equivalent to obtaining the global optimum by Algorithm 4. Hence we present the computing time of the additive FPTAS in respect of $\sigma$, and then provide the resulting gap values.

Figure 6 presents the computing time of the FPTAS, and compares the values to those of Algorithm 4. For the two objectives min-max and min-sum, the latter requires higher computing effort; this is consistent with the difference in the time for running OPTLABEL $\left(k^{2.5} \log k\right.$ versus $k^{3}$, see Section 6). Setting $\sigma=2$, the number of labels to be treated is reduced by at least half. This effect can be observed in Figure 6. Moreover, letting $\sigma$ go 


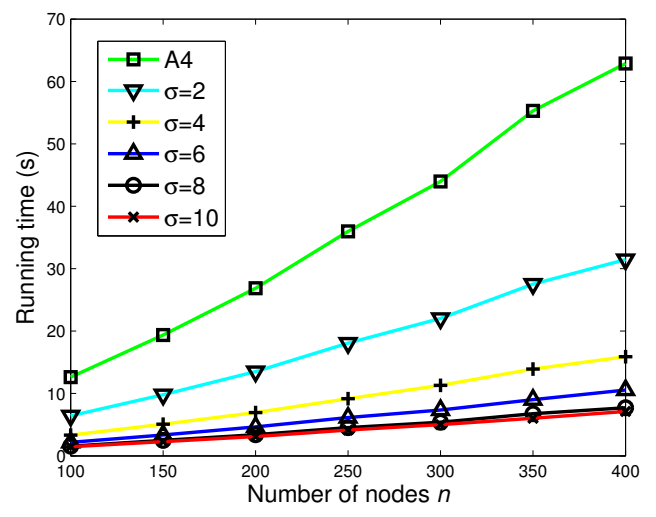

(a) $\min -\max$

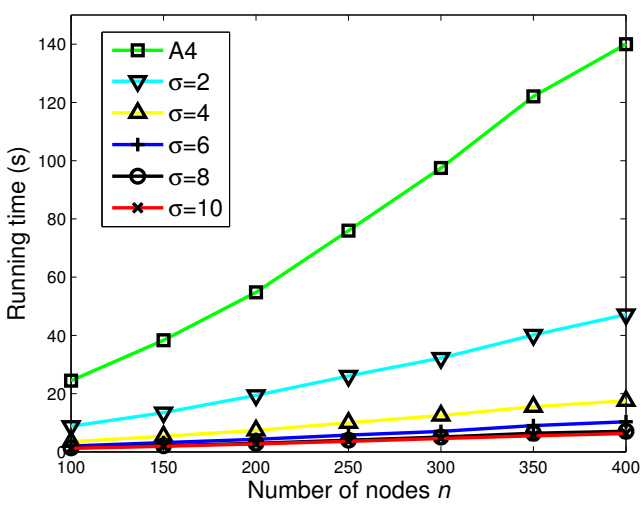

(b) min-sum

Figure 6: Computing time of the additive FPTAS in comparison to Algorithm 4.

beyond four does not yield any noticeable reduction in time. This is because the port gains have range $[-15,0] \mathrm{dB}$. With $\sigma=2$, there are thus nine possible values after discretization. The number goes down to five for $\sigma=4$, but only to four for $\sigma=6$. Both $\sigma=8$ and $\sigma=10$ lead to three possible values and therefore there should not be any further noticeable difference in computing effort, and this is indeed verified by the results in Figure 6.

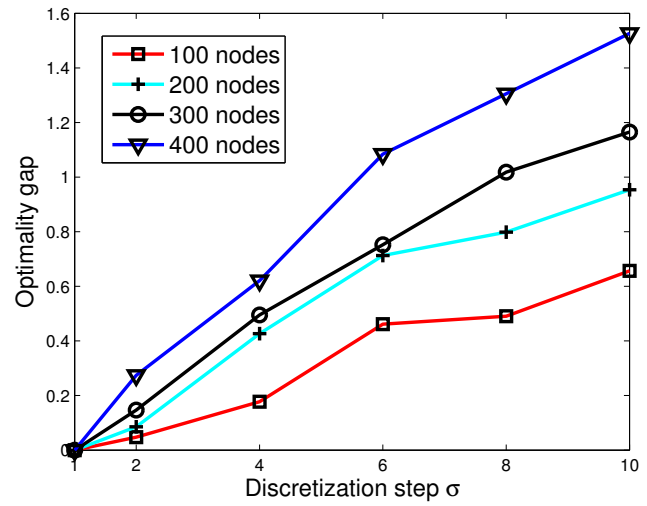

(a) $\min -\max$

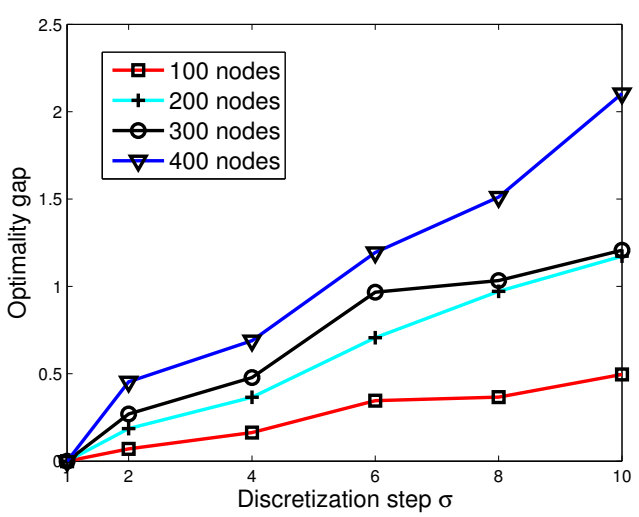

(b) min-sum

Figure 7: Empirical optimality gap of the additive FPTAS.

Both Algorithm 1 and Algorithm 4 have global optimality guarantee. In the next part of performance evaluation, we examine the power deviation of the FPTAS scheme in relation to global optimum, i.e., the difference between the power deviation of FPTAS and that of global optimum, normalized by the latter. In Figure 7, we show this relative gap of the FPTAS 
in respect of the discretization step $\sigma$. Note that when $\sigma=1$, the FPTAS coincides with Algorithm 4 and hence the optimality gap in power deviation is zero, because the input is integer-valued. For larger $\sigma$, the increase in gap with respect to $\sigma$ exhibits approximatively a linear shape. This is expected because of the (roughly) linear relation between $\sigma$ and $\epsilon$. Moreover, the gap grows in network size, verifying the analysis in Section 6 that for the same value of $\sigma$, the additive precision $\epsilon$ that can be guaranteed is dependent on $n$ (min-max) and $n^{2}$ (min-sum). By the same analysis, the gap for min-sum should be empirically higher than that for min-max, as confirmed by the figure.

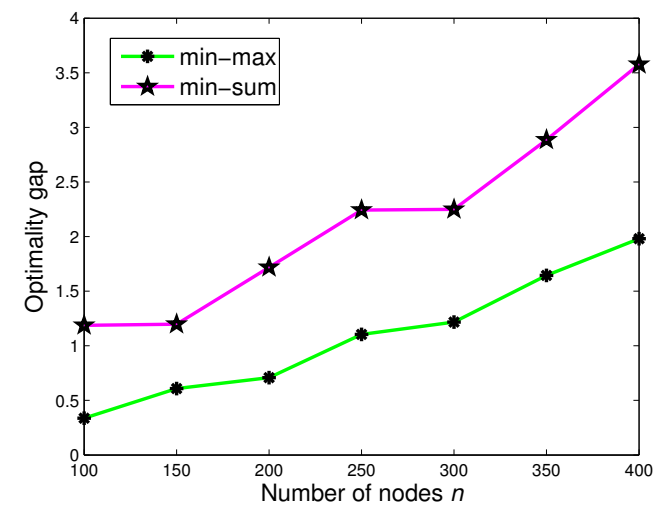

Figure 8: Optimality gap of the greedy algorithm.

As the last part of performance evaluation, we consider the greedy algorithm in Section 7 and show its optimality gap in Figure 8. In comparison to the FPTAS (cf. Figure 7(a) and Figure $7(\mathrm{~b})$ ), the greedy algorithm has comparable performance only for min-max and if the coarsest step size is used in the FPTAS. In all other cases, the greedy algorithm is clearly inferior, thus justifying the development of the proposed approximation scheme.

\section{Cost Considerations}

In this section we consider min-max and min-sum with equipment cost. For each node $v \in V_{I}$, let $c(g) \in \mathbb{N}$ denote the cost of the compatible equipment type $g \in G_{v}$. One way to address the cost aspect is to introduce a budget $B \in \mathbb{N}$ for equipment installation; thus a solution is 
feasible if $\sum_{v \in V_{I}} c\left(g_{v}\right) \leq B$.

- Algorithm 1 can be modified to account for a budget constraint as follows. A label $\ell$ is expanded, for both min-max and min-sum, to contain an additional component, representing the equipment cost incurred in the subtree. The cost is obviously zero at all leaf nodes. At an inner node $v \in V_{I}$, the function CombineLabels returns a label of which the cost equals the sum of the cost in each label $\ell_{w}$ for $w \in N_{v}$, plus that of the equipment type in question. This label is added to $L_{v}^{\prime}$ only if the cost does not exceed $B$. The label selection phase for constructing $L_{v}$ is adapted as well. Specifically, choosing an optimal label is conducted for each potential power $p \in \Omega$ for $v$ as well as each $\beta \in \Omega$ for $T_{v}$, that is, $\operatorname{argmin}_{\ell \in L_{v}^{\prime}} f_{\ell}(p)$ is computed subject to a total cost of at most $\beta$. As the worst-case number of labels in $L_{v}$ increases to $B U$, the running time becomes $O\left(n\left(m k !(B U)^{k} c_{1}+(B U)^{2} c_{2}\right)\right)$, where $k, c_{1}$, and $c_{2}$ are defined as in Theorem 2 .

- For Algorithm 4, dealing with a budget constraint is more involved. In fact, applying the algorithm requires a slight violation (and hence relaxation) of the budget constraint. More precisely, for any desired accuracy $\epsilon>0$, there exists a randomized pseudopolynomial-time algorithm that returns an equipment solution performing not worse than $(1+\epsilon)$ times the optimum, using a budget of at most $(1+\epsilon) B$. Toward these ends, the routine OptLABEL has to solve a budgeted linear assignment (or bottleneck linear assignment) problem on a multi-graph. This problem amounts to selecting an assignment of children to ports as well as a label for each children so as to minimize the objective while satisfying the budget. The multi-graph stems from the fact that, for each port-child pair, the children's labels give rise to parallel edges with different objective function contributions and equipment costs. This problem can be transformed to finding a perfect matching in a bipartite graph subject to a budget constraint, a problem for which there exists a randomized FPTAS [6, 11, 21].

- The underlying idea of the FPTAS in Section 6 is easily extended to obtain a solution 
of value at most $O P T+\epsilon U$ with equipment cost no greater than $(1+\epsilon) B$. If the budget constraint is strict, however, both min-max and min-sum become much harder to approximate, and we are not aware of results in line with those in Section 6.

The above extensions remain applicable for equipment re-optimizaton given new equipment types and cost considerations. For each inner node, the type of equipment currently installed is in the compatible equipment set of the node with cost zero. This problem, subject to a strict budget constraint, does admit an FPTAS if the equipment cost is equal for all the new equipments types, as in this case there is no need for discretization. However, if one is allowed to move the currently installed equipment from a node to another one (assuming it is compatible for both), the problem becomes significantly harder, and the algorithms are no longer applicable. In this case both min-max and min-sum become strongly NP-hard, which can be shown from a reduction from 3-PARTITION and, therefore, unless $\mathrm{P}=\mathrm{NP}$, there is neither a pseudopolynomial-time algorithm, nor an additive FPTAS.

\section{Conclusions}

We have considered a combinatorial optimization problem on a tree, where the node configurations along the path from the root to each leaf determine the performance value at the leaf, with the application context of equipment selection in passive distributed antenna systems. The objective function is to minimize the maximum or the total deviation from the specified target values at the leaves. We have shown that both min-max and min-sum are weakly NP-hard. The bulk of our algorithmic development leads to the following results. For global optimality, we have developed pseudopolynomial-time algorithms for the general problem setting as well as for trees with bounded node out-degree. Based on these algorithms, fully-polynomial-time additive approximation schemes are derived. These results settle the complexity status of both min-max and min-sum, in view of the fact that no polynomial approximation algorithm with a multiplicative approximation factor exists unless $\mathrm{P}=\mathrm{NP}$. 
In addition, we have provided algorithmic extensions to account for equipment cost budget.

The study is subject to several extensions. One line of research is to examine if the running time of the pseudopolynomial-time algorithms can be further improved by studying, for example, if extensions of the technique in [17] can be derived. A second interesting topic is formed by follow-up studies of min-max and min-sum with a cost budget. Another extension is to consider the capacitated version of the equipment selection problem, in which a maximum number of units is specified for each equipment type. Finally, the problem of joint topology and equipment selection optimization calls for further research.

\section{Acknowledgments}

The authors would like to thank the anonymous reviewers for their valuable comments and suggestions, and Fabrizio Grandoni for the helpful discussions on the budgeted matching. The work of the last author has been funded by the Linköping-Lund Excellence Center in Information Technology (ELLIIT), and European Marie Curie IRSES Project WiNDOW.

\section{References}

[1] T. Alade, H. Zhu, and H. Osman. Spectral efficiency analysis for distributed antenna system for in-building wireless communication. Proc. of IEEE PIMRC, pages 2075-2079, 2011.

[2] T. Alade, H. Zhu, and J. Wang. Uplink co-channel interference analysis and cancellation in femtocell based distributed antenna system. Proc. of IEEE ICC, pages 1-5, 2010.

[3] R. Atawia, M. Ashour, T. El Shabrawy, and H. Hammad. Optimized transmitted antenna power indoor planning using distributed antenna systems. Proc. of the 9th Wireless Communications and Mobile Computing Conference (IWCMC), pages 993-1000, 2013. 
[4] A. Attar, H. Li, and V. C. M. Leung. Green last mile: how fiber-connected massively distributed antenna systems can save energy. IEEE Wireless Communication Magazine, 18(5):66-74, 2011.

[5] H. Beijner. The importance of in-building solutions in third generation networks. Technical report, Ericsson Review No.2, 2004.

[6] A. Berger, V. Bonifaci, F. Grandoni, and G. Schäfer. Budgeted matching and budgeted matroid intersection via the gasoline puzzle. Mathematical Programming, 128:355-372, 2011.

[7] J. Borkowski, J. Niemelä, T. Isotalo, P. Lähdekorpi, and J. Lempiäinen. Utilization of an indoor DAS for repeater deployment in wcdma. Proc. of IEEE VTC Spring, pages 1112-1116, 2006.

[8] R. Burkard, M. Dell'Amico, and S. Martello. Assignment Problems. Society for Industrial and Applied Mathematics, 2009.

[9] L. Chen and D. Yuan. Mathematical modeling for optimal design of in-building distributed antenna systems. Computer Networks, 57(17):3428 - 3445, 2013.

[10] M. R. Garey and D. S. Johnson. Computers and Intractability: A Guide to the Theory of NP-Completeness. W. H. Freeman, 1979.

[11] F. Grandoni, R. Ravi, and M. Singh. Iterative rounding for multi-objective optimization problems. Proc. of European Symposium on Algorithms (ESA), pages 95-106, 2009.

[12] K. Hiltunen, B. Olin, and M. Lundevall. Using dedicated in-building systems to improve HSDPA indoor coverage and capacity. Proc. of IEEE VTC Spring, pages 2379-2383, 2005.

[13] D. Hong, S. Choi, and J. Cho. Coverage and capacity analysis for the multi-layer CDMA macro/indoor-picocells. Proc. of IEEE ICC, pages 354-358, 1999. 
[14] J. E. Hopcroft and R. M. Karp. An $n^{5 / 2}$ algorithm for maximum matchings in bipartite graphs. SIAM Journal on Computing, 2(4):225-231, 1973.

[15] RANPLAN iBuildNet ${ }^{\circledR}$. Ranplan. www.ranplan.co.uk, 2014.

[16] T. Isotalo and J. Lempiäinen. HSDPA Measurements for Indoor DAS. Proc. of IEEE VTC Spring, pages 1127-1130, 2007.

[17] D. S. Johnson and K. A. Niemi. On knapsacks, partition, and a new dynamic programming technique for trees. Mathematics of Operations Research, 8:1-14, 1983.

[18] R. Jonker and A. Volgenant. A shortest augmenting path algorithm for dense and sparse linear assignment problems. Computing, 38(4):325-340, 1987.

[19] H. Osman, H. Zhu, and T. Alade. Deployment of distributed antenna aystems in high buildings. Proc. of IEEE VTC Spring, pages 1-5, 2011.

[20] H. Osman, H. Zhu, D. Toumpakaris, and J. Wang. Achievable rate evaluation of inbuilding distributed antenna systems. IEEE Transactions on Wireless Communications, $12(7): 3510-3521,2013$.

[21] C. Papadimitriou and M. Yannakakis. On the approximability of trade-offs and optimal access of web sources. Proc. of the 41st IEEE Symposium on Foundations of Computer Science, pages 86-92, 2000.

[22] J. Perez-Rornero, O. Salient, and R. Agusti. On the capacity degradation in W-CDMA uplink/downlink due to indoor traffic. Proc. of IEEE VTC Fall, pages 856-859, 2004.

[23] B. Song, R. Cruz, and B. Rao. Downlink optimization of indoor wireless networks using multiple antenna systems. Proc. of IEEE INFOCOM, pages 2778-2789, 2004.

[24] T. Sorensen and P. Mogensen. Radio channel measurements on an eight-branch indoor office distributedantenna system. Proc. of IEEE VTC Spring, pages 328-332, 2001. 
[25] M. Tolstrup. Indoor Radio Planning: A Practical Guide for GSM, DCS, UMTS and HSPA. John Wiley \& Sons, 2 edition, 2011.

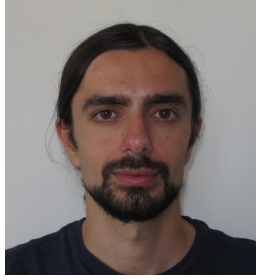

David Adjiashvili received the B.Sc. degree in Computer Science from the Tel-Aviv University, Israel in 2005, and his M.Sc. degree in Computer Science and Applied Mathematics from the Weizmann Institute of Science, Israel in 2007. He obtained a Ph.D. in Mathematics from ETH Zürich, Switzerland in 2012. He has since been a post-doctoral researcher at ETH Zürich at the Institute of Operations Research (IFOR). His research includes approximation algorithms for NP-hard problems, robust optimization, mathematical optimization, packing and scheduling problems.

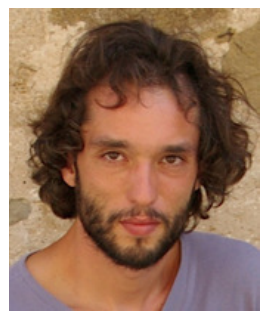
Sandro Bosio received his M.Sc. in Computer Science from Università degli Studi di Milano in 2002, and his Ph.D. in Mathematical Engineering from Politecnico di Milano in 2006. Between 2006 and 2012 he has been postdoc at Politecnico di Milano, Magdeburg Universität, and ETH Zürich. Since 2013 he is a consultant at Quintiq, an industry leader in supply chain planning and optimization. His research interests include complexity theory and mathematical optimization, in particular for covering and packing problems.

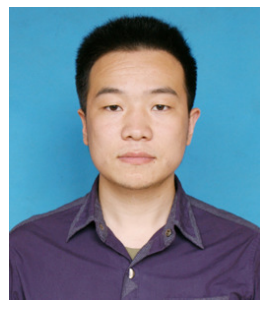

Yuan Li received his BSc and MSc degrees in Computer Science and Engineering in 2007 and 2009 respectively, both at the National University of Defense Technology (NUDT), China. He is currently working toward a Ph.D. degree at the Department of Electrical and Information Technology, Lund University, Sweden. His research interests span network modeling, algorithms, integer programming and other combinatorial methods with applications to communication networks.

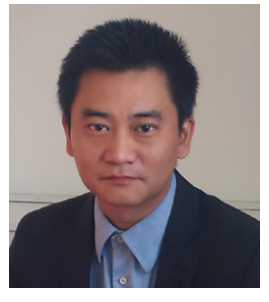

Di Yuan received his MSc degree in Computer Science and Engineering, and PhD degree in operations research at Linköping University, Sweden, in 1996 and 2001, respectively. At present he is full professor in telecommunications at the Department of Science and Technology, Linköping University, and head of a group in mobile telecommunications. Dr Yuan has been part time with Ericsson Research, 
Sweden, in 2011 and 2013. He has been the Principal Investigator of five European FP7 grants. He is a co-recipient of IEEE ICC '12 Best Paper Award. 